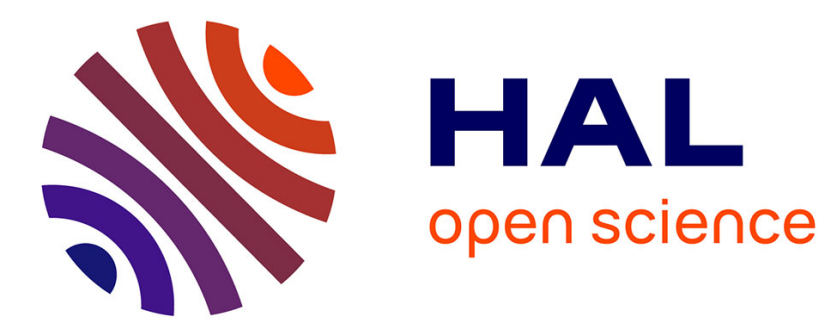

\title{
Electromagnetic glitch on the AES round counter
}

Amine Dehbaoui, Amir-Pasha Mirbaha, Nicolas Moro, Jean-Max Dutertre, Assia Tria

\section{To cite this version:}

Amine Dehbaoui, Amir-Pasha Mirbaha, Nicolas Moro, Jean-Max Dutertre, Assia Tria. Electromagnetic glitch on the AES round counter. Fourth International Workshop on Constructive Side-Channel Analysis and Secure Design - COSADE'2013, Mar 2013, Paris, France. pp 17-31, 10.1007/978-3-64240026-1 . emse-00837514

\section{HAL Id: emse-00837514 https://hal-emse.ccsd.cnrs.fr/emse-00837514}

Submitted on 24 Feb 2014

HAL is a multi-disciplinary open access archive for the deposit and dissemination of scientific research documents, whether they are published or not. The documents may come from teaching and research institutions in France or abroad, or from public or private research centers.
L'archive ouverte pluridisciplinaire HAL, est destinée au dépôt et à la diffusion de documents scientifiques de niveau recherche, publiés ou non, émanant des établissements d'enseignement et de recherche français ou étrangers, des laboratoires publics ou privés. 


\title{
Electromagnetic Glitch on the AES Round Counter
}

\author{
Amine Dehbaoui ${ }^{1}$, Amir-Pasha Mirbaha ${ }^{2}$, Nicolas Moro ${ }^{1}$, \\ Jean-Max Dutertre ${ }^{2}$ and Assia Tria ${ }^{1}$ \\ 1 CEA-LETI \\ firstname.lastname@cea.fr \\ 2 École nationale supérieure des Mines de Saint-Étienne \\ lastname@emse.fr \\ Département SAS - Systèmes et Architectures Sécurisés \\ Centre Microélectronique de Provence-Georges Charpak \\ 880 Avenue de Mimet, F-13541 Gardanne, France
}

\begin{abstract}
This article presents a Round Addition Analysis on a software implementation of the Advanced Encryption Standard (AES) algorithm. The round keys are computed on-the-fly during each encryption. A non-invasive transient fault injection is achieved on the AES round counter. The attack is performed by injecting a very short electromagnetic glitch on a 32-bit microcontroller based on the ARM Cortex-M3 processor. Using this experimental setup, we are able to disrupt the round counter increment at the end of the penultimate round and execute one additional round. This faulty execution enables us to recover the encryption key with only two pairs of corresponding correct and faulty ciphertexts.
\end{abstract}

\section{Introduction}

A fault in a cryptographic system refers to an accidental or an intentional disturbance that causes the encryption process to deviate from its correct execution or result. In this case, the cryptographic system may act abnormally or the result of encryption (or decryption) may be incorrect, considered as faulty.

The first alert about the feasibility of using faults to break cryptosystems was reported by D. Boneh et al. in [7. A Fault Attack consists in using hardware malfunction to infer secrets from the target's faulty behavior or output. The fault injection can be performed by various physical perturbation techniques, as reported in [5]. The first structured method for exploiting the secrets from faulty encryptions was presented as Differential Fault Analysis (DFA) in [6]. Since

The final publication is available at Springer via http://dx.doi.org/10.1007/978-3642-40026-1_2 
then, more analysis methods have been developed to reveal secrets from faulty behavior or outputs.

Among different perturbation methods, creating strong electromagnetic (EM) disturbances on the top of a circuit is a practical way to induce faults. J.-J. Quisquater and D. Samyde reported in [15] the possibility of an EM fault injection into the transistors and into the memory cells of a smart-card circuit. Afterwards, other research, e.g. [17] and [9, reported further successful EM fault attacks on cryptosystems.

In this paper, we introduce a new round addition attack induced by ElectroMagnetic Glitch (EMG) injection on an up-to-date microcontroller running an AES algorithm. The resulting erroneous ciphertexts are then processed in order to retrieve the secret key. This process involves cryptanalysis and differentiation techniques often used by the DFA.

This article is organized as follows: EMG fault injection is briefly presented in section 2 Then, the experimental setup, describing the targeted microcontroller and the EM attack bench, is presented in section 3. A quick reminder on the AES is given in section 4 . It is followed by the state-of-the-art of Round Modification Analysis and the proposed Round Addition attack. The experiment outline and the corresponding cryptanalysis are described in section 5 . To conclude, our findings are summarized in section 6 with further perspective.

\section{Electromagnetic Glitch Injection Technique}

Non-invasive fault injection techniques, such as EMG, represent a serious threat to the security of cryptographic circuits. They are sometimes considered as more dangerous than semi-invasive or invasive techniques because they do not require any chip decapsulation proficiency and equipment.

Choukri et al. showed in 8 the possibility of using power glitches to reduce the number of rounds of an AES implementation. Kim et al. also used power glitches in 11 to skip subroutine calls in a software RSA-CRT implementation. Similarly, Schmidt et al. [16 prevented a subroutine call in a square-and-multiply RSA software implementation. More recently Balasch et. al [4 performed a study of the clock glitch effects on a 8-bit AVR microcontroller. They showed that instructions can be replaced or skipped by injecting a clock glitch, and that the effects of faults are deterministic and reproducible. More precisely, as the clock period decreases, a larger number of the opcode's bits are stuck at zero.

The efficiency of EMG is mainly due to the inner properties of electromagnetic waves. Their ability to propagate through different materials is the most interesting one since it allows an attacker (without any preliminary preparation of the chip) to induce a very short glitch in the power supply voltage. This short glitch in the power supply voltage is the result of a coupling mechanism between the coil antenna and the targeted chip's Power Ground Network (PGN) 1419.

In comparison with power glitch, EMG technique allows the attacker to target a small part of the internal PGN by choosing an accurate XYZ stage and a small 
antenna diameter. A study about the EMG localized effect is presented in [9]. As reported by the authors, the propagation delays are increased through the circuit's logic when the EMG is injected on top of the die surface. Thus, by the violation of the circuit's timing constraints, the operations are not accomplished during the expected time and faults appear.

According to previous experiments in [9] on a basic 8-bit AVR microcontroller, an EMG induces faults during the program execution. A careful analysis of the faulty behavior, revealed that they were due to an instruction skip at the instant of the EMG injection. To the best of our knowledge, no EMG fault injection has been reported on an up-to-date 32-bit microcontroller. This fault model seems to be very threatening, since the opponent may be able to skip or to prevent a subroutine call, just by an EMG injection.

\section{Practical Electromagnetic Glitch Setup}

As described in section 2, EMG technique can be used to avoid the execution of an instruction on a microcontroller. In this section, the EMG injection setup used to generate transient EM pulses is described.

\subsection{EMG Platform}

The EMG platform depicted in Figure 1 is composed of a control computer, the target device, a motorized stage, a pulse generator, and a magnetic antenna. The target (described in subsection 3.2) is mounted on the XYZ motorized stage. The computer controls both the pulse generator (through a RS-232 link) and the target board (through a UsB link).

The pulse generator is used to deliver voltage pulses to the magnetic coil. It has a constant rise and fall transition time of $2 \mathrm{~ns}$. The amplitude range (respectively the width) of the generated pulses extends from -200V to $200 \mathrm{~V}$ (respectively from $10 \mathrm{~ns}$ to $200 \mathrm{~ns}$ ). We use a magnetic antenna composed of a few turns with a diameter of $1 \mathrm{~mm}$ in order to only disturb a small part of the targeted device. This spatial accuracy is possible thanks to a high accuracy XYZ stage.

\subsection{Target}

The chosen target is an up-to-date 32-bit microcontroller, designed in a CMOS $130 \mathrm{~nm}$ technology. It is based on the ARM Cortex-M3 processor [2]. Its operating frequency is set to $24 \mathrm{MHz}$.

Choice of the Target : For our target, we were looking for a state-of-the-art microchip, based on a recent technology. We chose an ARM Cortex-based microcontroller because ARM Cortex processors are already very widespread for both reasons of the mainstream and their security. Nowadays, more and more integrated circuit manufacturers propose ARM Cortex based microcontrollers. 
Although we did not choose a smart-card version of the microcontroller, our target embeds some security mechanisms against clock perturbations, voltage glitches and other kinds of hardware faults. Moreover, it enables the programmer to define some interrupts in order to handle some hardware exceptions triggered by the core. Hence, we can consider our target as reasonably secured against some of the most common low-cost fault injection means. However, there is no widespread countermeasure against electromagnetic injection. Thus, we assumed that it could be considered as an up-to-date realistic target to study the embedded security mechanisms and to perform electromagnetic glitch injection.

Architecture Details : The microcontroller embeds 128kB of flash program memory and 8KB of RAM. The core uses an ARMv7-M Harvard architecture and embeds a 3-stage pipeline. It is able to run both Thumb and Thumb-2 RISC instruction sets from ARM. Thumb-2 is an extension of the Thumb 16-bit instruction set

which $\mathrm{cl}$

tection

Hardwa:

Redund.

integrity

a specifi

interrup

security

execute

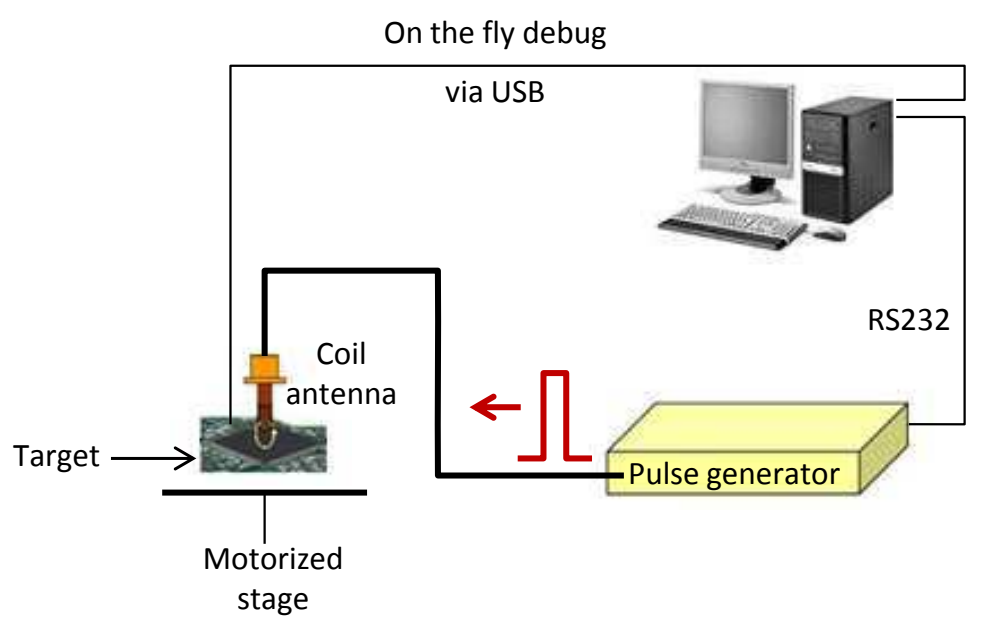

Fig. 1. Electromagnetic glitch platform 
Table 1. List of available hardware interrupts

\begin{tabular}{|l|l|}
\hline Exception & Description \\
\hline Hard fault & $\begin{array}{l}\text { Error during exception processing } \\
\text { Has the highest priority }\end{array}$ \\
\hline Bus fault & $\begin{array}{l}\text { Memory related fault } \\
\text { For an instruction or data memory transaction }\end{array}$ \\
\hline $\begin{array}{l}\text { Memory } \\
\text { Management Fault }\end{array}$ & $\begin{array}{l}\text { Triggered by the memory protection unit } \\
\text { Possible access to a restricted memory area }\end{array}$ \\
\hline Usage Fault & $\begin{array}{l}\text { Fault related to instruction execution } \\
\text { Undefined instruction, illegal unaligned access, etc. }\end{array}$ \\
\hline $\begin{array}{l}\text { Clock Security } \\
\text { System }\end{array}$ & Error on the high speed external clock \\
\hline $\begin{array}{l}\text { Programmable } \\
\text { Voltage Detect }\end{array}$ & The power supply is under a user-defined threshold \\
\hline
\end{tabular}

\subsection{EMG Impact on the Microcontroller Power Supply}

Before the logical effects of an EMG can be investigated, the EMG profile of the target must be established. Figure 2 shows the target's power supply during the EMG injection. This measurement was done using a differential probe with DC filtering. As we can observe, for a $180 \mathrm{~V}$ injected EMG during $20 \mathrm{~ns}$, we obtained a negative spike of less than $50 \mathrm{~ns}$ width and $300 \mathrm{mV}$ amplitude. These voltage variations may seem quite small especially in order to induce faults into the device computations. However, because the power supply measurement was done out of the core, a large part of the perturbation may have been filtered out.

\section{Round Modification Analysis on AES: State-of-the-Art}

\subsection{Advanced Encryption Standard}

The Advanced Encryption Standard, according to [12, is a symmetric block cipher that processes data blocks of 128 bits, using cipher keys with lengths of 128,192 , and 256 bits, respectively in 10, 12 or 14 rounds. For the sake of simplicity, we consider in this paper only the 128-bit AES version: denoted AES or AES-128.

AES has two separated processes: One for the KeyExpansion to derive round keys from the secret key and another one for the DataEncryption. AES-128 performs the encryption process in 10 rounds, after a short initial round. A round key is used during the computations of every round. Hereafter, we use the " $K$ " prefix plus the round number to refer to a round key (e.g. " $K_{9}$ " for the $9^{t h}$ round key).

To encrypt a plaintext, namely $M$, the encryption process considers its 16 bytes as a matrix of $4 \times 4$ bytes. Each round of the algorithm, except the initial and the last ones, includes 4 transformations: First, the value of each matrix 
element, i.e. one byte value, is exchanged with the corresponding value in a substitution table (SubBytes or SB). Secondly, a rotational operation on the matrix rows is executed (ShiftRows or SR). In the third step, the algorithm applies a linear transformation to each element and combines it with other values of the same column using a different coefficient of 1,2 or 3 for each element (MixColumns or MC) in $\operatorname{GF}\left(2^{8}\right)$. The fourth operation is a bitwise xor (AddRoundKey or ARK) is performed between the value of each element and the corresponding byte of the round key (RoundKey or $K_{R C}$ ). Before the first round, an ARK is applied to $M$ and $K$ (i.e. Round 0 ). The MC transformation is omitted in the last round.

The AES algorithm takes the key $K$ and performs a KeyExpansion routine to generate a key schedule. The KeyExpansion generates a total of $R_{\max }$ round keys. KeyScheduling (KS) is a set of linear and non-linear transformations that calculates a new round key from the previous one. The initial round key, $K_{0}$, is equal to $K$. Each of the following round keys (i.e. $K_{1}, K_{1} \ldots K_{R_{\max }}$ ) is derived from the previous one.

Algorithm 1 shows an AES DataEncryption implementation which has many similarities with the official AES specifications [12. In this algorithm 1. $C$ is an intermediate variable used to memorize the AES state throughout the encryption process. The round counter, hereafter $R C$, is used as an index to select the corresponding round key during each ARK transformation. Moreover, $R C$ is compared

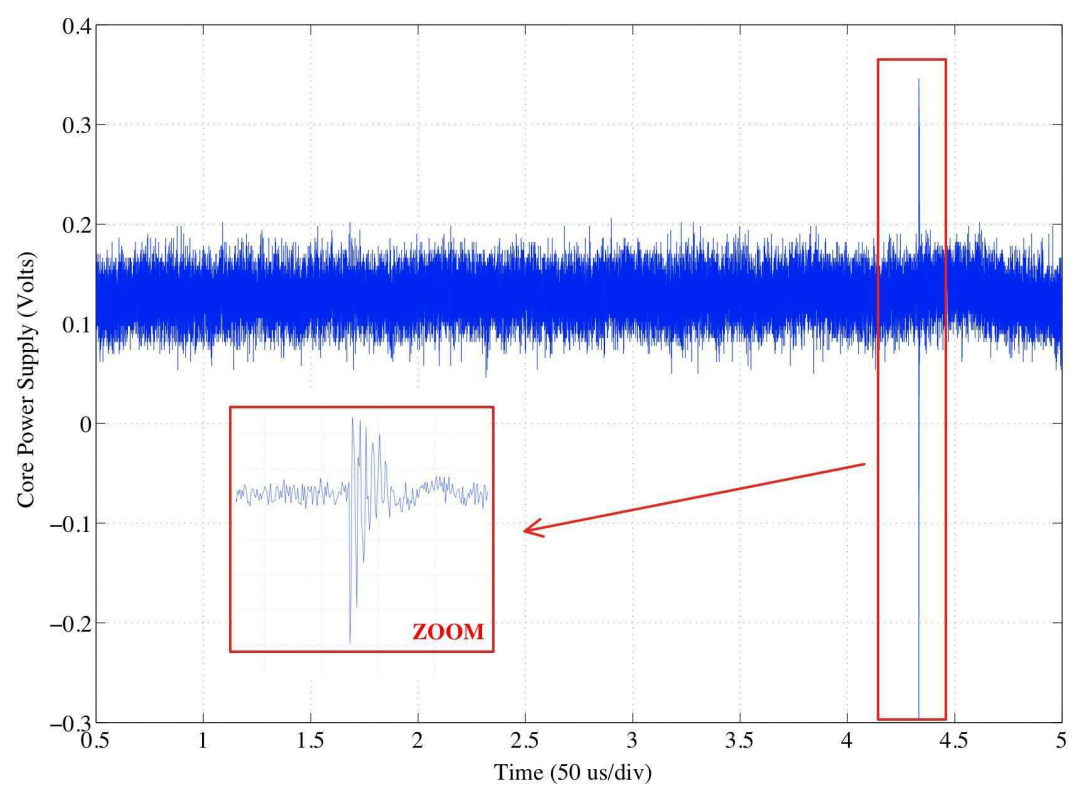

Fig. 2. EMG impact on the microcontroller power supply during AES execution 
to the total round number reference, $R_{\max }$, to end the iterative loop preceding the final round.

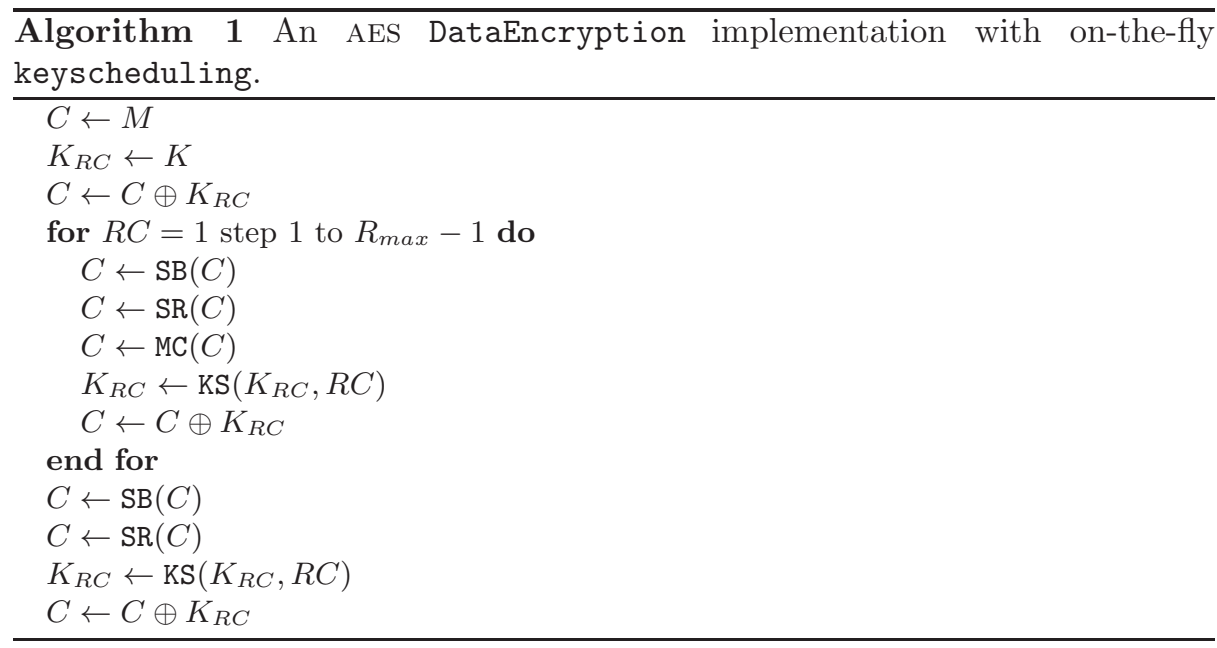

According to the algorithm purposes and the circuit resources, various options for KeyExpansion implementation are possible. The KeyExpansion may be executed only one time after a circuit reset or at the beginning of algorithm execution. Thus, the calculated round keys must be stored in the memory for any further encryption. The opposite solution is to calculate the round keys on-thefly for each encryption. The proposed AES implementation, shown as algorithm 1 calculates each round key on-the-fly at its corresponding round.

A significant part of AES algorithm strength against cryptanalysis is based on its repeated rounds. Any modification in the number of AES rounds may reduce the cipher's security 10. This kind of attacks was previously reported in some research experiments. We refer the readers to the next subsection.

\subsection{Previous Round Modification Analysis Attacks}

In 2005, H. Choukri and M. Tunstall reported in 8 the shortening of the AES execution to only one round (after the initial round) by fault injection and thus finding the key. 10 illustrated that it is also possible to increase or to alter the AES rounds by fault injection and then to discover the key.

Round Modification Analysis principle is based on decreasing or increasing the number of rounds or altering their execution in an algorithm in order to facilitate subsequent cryptanalysis 10. For instance, let's consider an attack that makes a jump, after executing a few instructions or the first round at the beginning of algorithm, to its end. The remaining encryption processes are thus skipped. Therefore, the final ciphertext is the product of fewer algorithm 
processes that may reveal the key more easily. Besides, an attack that adds or removes only one or two rounds of a normal encryption may permit a differential analysis by using unmodified encryptions as the reference.

Notation: In the following, we use the " $R$ " prefix plus the round number to refer to the transformations involved in an AES round. Hence, $R_{0}-R_{1}-R_{2}-R_{3}-R_{4^{-}}$ $R_{5}-R_{6}-R_{7}-R_{8}-R_{9}-R_{10}$, or shortly $R_{0} \ldots R_{10}$, represents the rounds of a complete (i.e. unmodified) AES. " $M_{i}$ " represents the AES intermediate state at the end of round $i$. We use $R_{m=j}$ to express that, due to a fault, a round composed of the $\mathrm{ARK} \circ \mathrm{MC} \circ \mathrm{SR} \circ \mathrm{SB}$ transformations (where " $m$ " stands for middle round) is using an incorrect round key of index $j$. Note that $j$ may be higher than the number of rounds. $R_{f=j}$ has the same meaning for a round without the MC transformation ( " $f$ " stands for final round).

Here, we present briefly the state-of-the-art of previous Round Modification Attacks on AES:

H. Choukri and M. Tunstall's Attack They showed in [8] that a transient glitch on the power supply of a microcontroller may change the $R C$ value of an iterative cipher. If the opponent changes the $R C$ of an AES program at the beginning of algorithm execution to its final value, the ciphertext will be the product of a single executed round (plus the initial round): $R_{0}-R_{m}$ or $R_{0}-R_{f}$ (according to the notation introduced in section 4.1).

Thus, the cryptanalysis of this very short encryption process does not correspond anymore to the complexity of a correct AES execution that includes 10 rounds. 8] introduced a cryptanalysis technique that makes it possible to retrieve the secret key. This technique obtains eq. 1 by xoring two faulty outputs, $D^{a}$ and $D^{b}$ ( $M^{a}$ and $M^{b}$ being the corresponding plaintexts):

$$
\mathrm{MC}^{-1}\left(D^{a} \oplus D^{b}\right)=\mathrm{SB}\left(M^{a} \oplus K\right) \oplus \mathrm{SB}\left(M^{b} \oplus K\right)
$$

For every key byte, Eq. 1 yyields two different hypotheses. Finally, an exhaustive search over the $2^{16}$ possible keys is made to retrieve the secret key. Note that this cryptanalysis does not require any knowledge of the correct encryptions for $M^{a}$ and $M^{b}$.

J.H. Park et al.'s Attack They reported in [13] a laser fault injection on an ATmega128 8-bit microcontroller which embeds an AES. The algorithm implementation is compliant with the algorithm structure proposed in [12].

They described a successful attack that consists in jumping from $R_{1}$ to $R_{10}$. The faulty execution path is $R_{0}-R_{1}-R_{10}$. Therefore, an additional round is executed in comparison to [8] that includes only $R_{0}-R_{m}$ (or $R_{0}-R_{f}$ ).

The associated cryptanalysis requires data from ten different reduced encryptions. Calculations involve four steps of exhaustive search of $2^{40}, 2^{32}, 2^{24}$, and $2^{32}$ steps respectively. This takes approximately ten hours on a PC. 
K.S. Bae et al.'s Attack They presented in [3] a successful attack by eliminating the AES penultimate round. The encryption includes $R_{0} \ldots R_{8}-R_{10}$. This attack is done by laser fault injection on an ATmega128 8-bit microcontroller which embeds an AES. The key is revealed using two pairs of corresponding faulty and correct ciphertexts and then an exhaustive search between the two candidates for each key byte. Therefore, the cryptanalysis needs finding a key between $2^{16}$ values which has a computational complexity similar to the attack reported by H. Choukri and M. Tunstall.

J.M. Dutertre et al.'s Attack They showed in 10 three laser fault injection attacks, targeting either the round counter or the total round number reference of an AES. The first attack reduces the AES penultimate round and executes a 9-round AES. The second attack is based on the alteration of the round index during the penultimate and the final rounds. It changes the round key values in AddRoundKey but does not change the total number of executed rounds. Therefore, the encryption uses corrupted keys at the penultimate and at the final rounds. The key is revealed by using a differential analysis over three pairs of corresponding faulty and correct ciphertexts.

In their third experiment, they reported a Round Addition attack by targeting the total round number reference. The encryption performs $R_{0} \ldots R_{9}-R_{m=10^{-}}$ $R_{f=11}$. It uses the correct $K_{10}$ for the $10^{t h}$ intermediate round. Nevertheless, the final AddRoundKey is performed using a block of unknown values as $K_{f=11}$. The attack is exploited by using three pairs of corresponding faulty and correct ciphertexts through a differential analysis.

These attacks are reported successful on a 8-bit $0.35 \mu \mathrm{m}$ RISC microcontroller. [10] proposed to expand the category of "Round Reduction" in the Fault Attacks into "Round Modification Analysis" which covers a larger domain of algorithm modification attacks, by including the "Round Addition" and the "Round Alteration".

\subsection{Our Proposed Round Addition Attack}

In the third Dutertre et al.'s attack, reported in [10, injecting a fault equal to 0x01 into $R_{\max }$, at anytime before the end of $R_{9}$, lengthens the intermediate rounds by one round. Therefore a total of 11 rounds is executed. This attack was performed by a surgical laser fault injection to an algorithm almost similar to algorithm 1 (but with pre-calculated round keys). In our research, presented in this paper, we examined the feasibility of a similar Round Addition attack on the AEs by an EM glitch.

To meet this requirement, a solution may be an EMG attack on the $R C$ incrementation instruction at the end of $R_{9}$. Therefore, the $R C$ incrementation instruction may be skipped, due to the EMG effect on the MCU. Thus, $R C$ value remains 9 and another intermediate round, denoted $R_{9}^{\prime}$, is executed. 
In an AES implementation, similar to the Algorithm 1 each round key is calculated on-the-fly at its corresponding round. The KeyScheduling process is a function of the previous round key and the current index of the $R C$. Therefore, for the redundant $R_{m=9}^{\prime}$, a new round key (i.e. $K_{9}^{\prime}$ ) is derived from the previous one, i.e. $K_{9}$. In the same way, another invalid key value is derived from $K_{9}^{\prime}$ for the $R_{f=10}$, denoted as $K_{10}^{\prime}$.

Finally, the encryption sequence may be: $R_{0}-R_{1} \ldots R_{9}-R_{9}^{\prime}-R_{10}^{\prime}$, including a total of 11 executed rounds. In this case, the success of the attack requires a differential cryptanalysis distinct from the technique reported in [10.

In the AES algorithm, each middle round is composed of the $\mathrm{ARK} \circ \mathrm{MC} \circ \mathrm{SR} \circ \mathrm{SB}$ transformations. The final round does not include the MixColumns transformation. For the ease of writing the equations, we denote the final round transformations before the ARK by the FinalRound or the FR operation, described in Eq. 2

$$
\mathrm{FR}\left[M_{i+1}\right]=\mathrm{SR} \circ \mathrm{SB}\left[M_{i}\right]
$$

We also denote the middle round transformations before the ARK by the MiddleRound or the MR operation, described in eq. 3

$$
\operatorname{MR}\left[M_{i+1}\right]=\operatorname{MC} \circ \mathrm{SR} \circ \mathrm{SB}\left[M_{i}\right]
$$

The cryptanalysis of our proposed attack scheme requires only two pairs of correct and faulty ciphertexts $\left(C^{a}, D^{a}\right),\left(C^{b}, D^{b}\right)$. Considering two pairs of corresponding faulty and correct encryptions, we have:

$$
\begin{gathered}
C^{a}=\mathrm{FR}\left[M_{9}^{a}\right] \oplus K_{10} \\
C^{b}=\mathrm{FR}\left[M_{9}^{b}\right] \oplus K_{10} \\
D^{a}=\mathrm{FR}\left[\operatorname{MR}\left[M_{9}^{a}\right] \oplus K_{9}^{\prime}\right] \oplus K_{10}^{\prime} \\
D^{b}=\mathrm{FR}\left[\operatorname{MR}\left[M_{9}^{b}\right] \oplus K_{9}^{\prime}\right] \oplus K_{10}^{\prime}
\end{gathered}
$$

By combining Eq. 田and 5, and with an extra MC operation, we get :

$$
\operatorname{MR}\left[M_{9}^{a}\right] \oplus \operatorname{MR}\left[M_{9}^{b}\right]=\operatorname{MC}\left[C^{a} \oplus C^{b}\right]
$$

In a similar way, by combining Eq. 6 and 7 , we get Eq. 9 where $K_{9}^{\prime}$ is removed:

$$
\mathrm{FR}^{-1}\left[D^{a} \oplus K_{10}^{\prime}\right] \oplus \mathrm{FR}^{-1}\left[D^{b} \oplus K_{10}^{\prime}\right]=\operatorname{MR}\left[M_{9}^{a}\right] \oplus \operatorname{MR}\left[M_{9}^{b}\right]
$$

Then by combining Eq. 8 and 9 we obtain :

$$
\mathrm{FR}^{-1}\left[D^{a} \oplus K_{10}^{\prime}\right] \oplus \mathrm{FR}^{-1}\left[D^{b} \oplus K_{10}^{\prime}\right]=\mathrm{MC}\left[C^{a} \oplus C^{b}\right]
$$


Since $C^{a}, C^{b}, D^{a}$ and $D^{b}$ are known values, Eq. 10 can be resolved by performing an exhaustive search over each of $K_{10}^{\prime}$ byte. This exhaustive search leads to 2 hypotheses for each of $K_{10}^{\prime}$ bytes. $K_{10}^{\prime}$ and $K_{9}^{\prime}$ are calculated using a correct, but redundant sequence of KeyScheduling. So, a second exhaustive search among $2^{16}$ whole-key hypotheses is necessary in order to obtain a unique value for each byte of $K_{10}^{\prime}$. Therefore, $K_{9}^{\prime}$ and consequently $K$ are recovered by simply putting $K_{10}^{\prime}$ through the inverse of KeyScheduling.

\section{$5 \quad$ Experimental Results}

In this section we describe a practical EMG injection into the instruction corresponding to the AES round counter incrementation. It results in the execution of a second $9^{\text {th }}$ round denoted $R_{9}^{\prime}$ (as described in the previous section).

\subsection{Experimental Outline}

A software version of the AES algorithm has been implemented on the microcontroller. This version provides AES subroutines with an "on-the-fly" KeyScheduling. The encryption process is written in C code. Its structure is given in Algorithm 1. We compile this source code using Keil MDK-ARM toolchain.

To monitor our microcontroller and our injection bench, we used a computer application. This application communicates with our microcontroller by using a Serial Wire Debug (SWD) interface. This interface is a non-intrusive 2pin alternative JTAG debug interface [1]. The experimental process used for our experiments is described in algorithm 2 .

The position of the probe on the top of the circuit's surface, influences the fault occurrence rate. We define the probe position by using a simple trial and error empirical method.

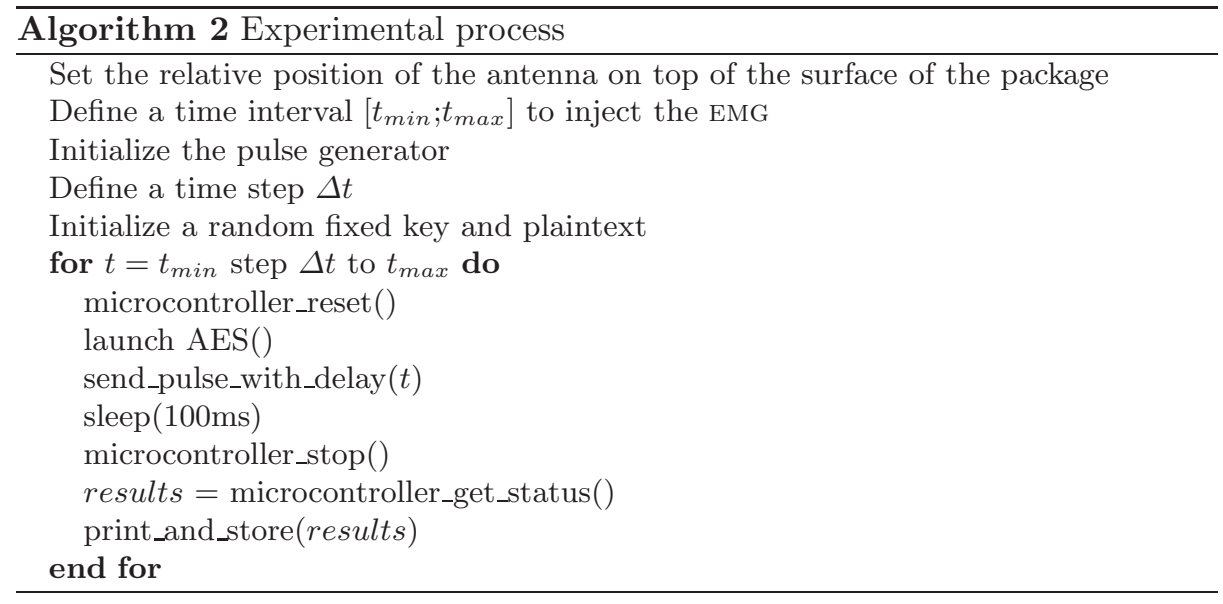


The microcontroller_get_status() function at the end of the AES computation displays the registers' values, the 16-byte ciphertext's value and the interrupt status flags.

For our experiments, we used a 100ps $\Delta t$ resolution. The sleep (100ms) enables us to be sure that the encryption will be finished when the microcontroller is stopped. After $100 \mathrm{~ms}$, we know that the microcontroller is in one of the following states :

- Running the infinite loop at the end of the AES computation

- Running the infinite loop of an interrupt (as detailed in section 3.2)

- Crashed

\subsection{Results}

Our aim was to disrupt the instruction that increments the round counter. Thus, we targeted a 500ns time interval between the $9^{\text {th }}$ and the $10^{\text {th }}$ rounds of the AES computation. For each encryption, we changed the pulse injection time. During the experiment, the EMG injection time spanned the entire time interval, from the beginning to the end, by steps of 100ps.

For each of these time steps, 100 encryptions of the same key and plaintext were carried out. The different types of EMG impacts in this experiment are reported in Fig. 3. Due to the large number of samples we got, we decided to plot a $5 \mathrm{~ns}$ interval. We chose a time interval during which the AES round counter was faulted.

At the beginning of the experimental process, we performed a first calibration step. This first execution enabled us to get the normal internal register and output values without any EMG injection. In Fig. 3ta, we got a fault when the output value was different from the one from our calibration execution. The Yaxis of Fig. 3-a represents the values of internal registers (R0-R12, SP, LR, PC, XPSR $)$ and the final value of the round counter $(r c=10)$. On this figure, green squares represent faults on the internal registers while red squares represent faults on the round counter.

Some of the faulted values mentioned on the graph are actually artifacts of our experiments, due to our experimental process. In the case that an interrupt subroutine is called, many registers are changed in the new stack frame. Thus, their values are different from the calibration execution. However, this is not directly due to EMG injection and cannot really be considered as a fault.

According to this timing cartography, our fault injection technique based on EMG injection close to a circuit enables to easily target the AES round counter incrementation instruction. More precisely we observed that the AES round counter has been faulted for different injection times, but in a very small time interval [17.2ns $-18.8 \mathrm{~ns}]$. We also observed that, in the event that no interrupt is raised, the internal CPU register R3 was the only corrupted value.

Figure 3-b reports the fault occurrence rate as a function of time. As we can observe, a curve that seems to look like a gaussian curve is obtained. This curve is 
centered around the interval in which the AES round counter was corrupted. The gaussian peak's center corresponds to the interval's center. For a time interval, the fault occurrence rate is equal to $100 \%$. 
Bus fault
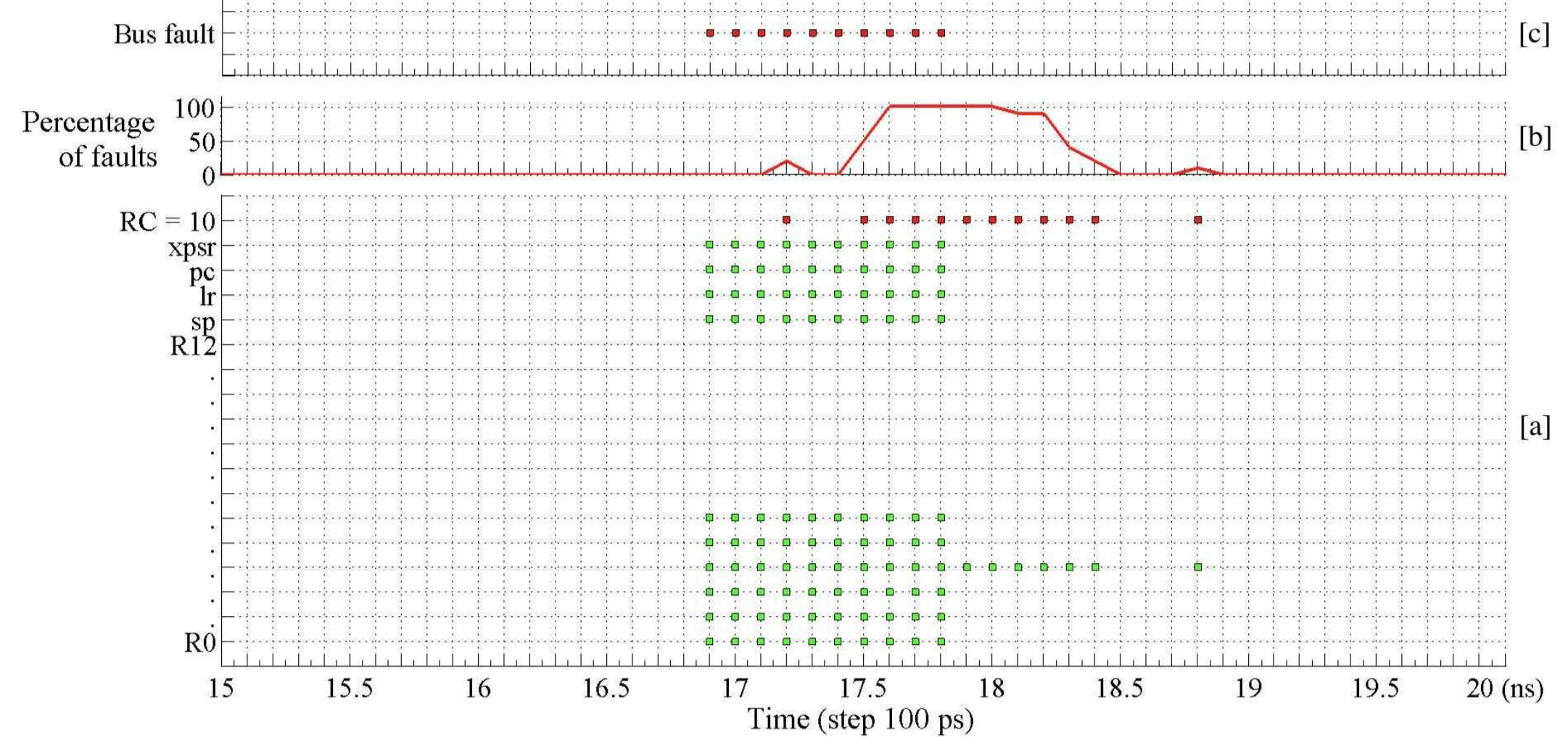

Fig. 3. Timing cartography of the EMG effect on the microcontroller 
Figure 3-c reports the timing interval where the microcontroller was able to detect the EMG and then raises its associated interrupt. In our case, the "Bus fault" exception was the only one triggered during our experiment. This exception indicates that a fault for an instruction or data memory transaction is detected. As we can observe, the interrupts were localized in [16.9ns-17.8ns]. Considering the time interval [17.9ns -18.8ns], we were able to perform the proposed attack without being detected by the microcontroller.

As we lack information about the microcontroller's design, it is very tough for us to precisely know the impact of the EMG inside the microcontroller. However, at a macroscopic level, we are able to sort the impacts into :

- Nothing happens

- A fault is produced and an interrupt is raised: [16.9ns -17.8ns]

- A fault is produced, no interrupt is raised, an assembly instruction is skipped: [17.9ns $-18.8 \mathrm{~ns}]$

\section{Conclusion}

In this paper we presented a new Round Addition attack on a software implementation of the Advanced Encryption Standard (AES) algorithm. The proposed attack consists in targeting the round counter $R C$ in order to induce the execution of a second penultimate round. The fault is induced at the end of the penultimate round during the incrementation of $R C$. EMG injection enables us to get effects that are equivalent, at a macroscopic level, to an instruction skip with a high occurrence rate and without triggering hardware interrupts. The proposed attack is achieved by skipping the counter increment instruction. As a result the faulty AES executes 11 rounds and enables us to recover the encryption key with only two pairs of corresponding correct and faulty ciphertexts.

Future works: According to our experimental results and at a macroscopic level, this instruction skip fault model was used to describe an attack model. However, the faults induced by EMG injection are probably more complex than an instruction skip and our fault model can be completed by using future experimental results. For further studies, we will also try to improve the way we choose our probe position.

\section{Acknowledgment}

This work was funded by the EMAISeci Project (ANR-10-SEGI-0012). 


\section{References}

1. ARM. ARM debug interface v5.

2. ARM. Documentation about cortex-m3 processors.

3. K.S. Bae, S.J. Moon, D.H. Choi, Y.J. Choi, D. Choi, and J.C. Ha. Differential fault analysis on AES by round reduction. In Proceedings of ICCIT'2011, pages 607-612. IEEE, 2011.

4. Josep Balasch, Benedikt Gierlichs, and Ingrid Verbauwhede. An in-depth and black-box characterization of the effects of clock glitches on 8-bit MCUs. In Proceedings of FDTC'2011, pages 105 -114, 2011.

5. A. Barenghi, L. Breveglieri, I. Koren, and D. Naccache. Fault injection attacks on cryptographic devices: Theory, practice, and countermeasures. Proceedings of the IEEE, 2012.

6. Eli Biham and Adi Shamir. Differential fault analysis of secret key cryptosystems. In Proceedings of CRYPTO'1997, volume 1294 of LNCS, pages 513-525. SpringerVerlag, 1997.

7. Dan Boneh, Richard DeMillo, and Richard Lipton. On the importance of checking cryptographic protocols for faults. In Proceedings of EuroCrypt 1997, volume 1233 of LNCS, pages 37-51. Springer-Verlag, 1997.

8. Hamid Choukri and Michael Tunstall. Round reduction using faults. Proceedings of FDTC'2005, pages 13-24, 2005.

9. A. Dehbaoui, J.M. Dutertre, B. Robisson, and A. Tria. Electromagnetic transient faults injection on a hardware and a software implementations of AES. In Proceedings of FDTC'2012, pages 7-15. IEEE, 2012.

10. J.-M. Dutertre, A.-P. Mirbaha, D. Naccache, A.-L. Ribotta, A. Tria, and T. Vaschalde. Fault round modification analysis of the advanced encryption standard. In Proceedings of HOST'2012. IEEE, 2012.

11. Chong Kim and Jean-Jacques Quisquater. Fault attacks for CRT based RSA: New attacks, new results, and new countermeasures. In Proceedings of WISTP'200\%, volume 4462 of LNCS, pages 215-228. Springer Berlin / Heidelberg, 2007.

12. NIST. Announcing the Advanced Encryption Standard (AES). Federal Information Processing Standards Publication, n. 197, November 26, 2001.

13. JeaHoon Park, SangJae Moon, DooHo Choi, YouSung Kung, and JaeCheol Ha. Differential fault analysis for round-reduced AES by fault injection. ETRI Journal, 33(3):434-442, 2011.

14. F. Poucheret, K. Tobich, M. Lisart, B. Robisson, L. Chusseau, and P. Maurine. Local and direct EM injection of power into CMOS integrated circuits. In Proceedings of FDTC'2011. IEEE, 2011.

15. Jean-Jacques Quisquater and David Samyde. Eddy current for Magnetic Analysis with Active Sensor. In Proceedings of Esmart'2002, 2002.

16. J-M Schmidt and C Herbst. A practical fault attack on square and multiply. In Proceedings of FDTC'2008, pages 53-58, 2008.

17. Jörn-Marc Schmidt and Michael Hutter. Optical and EM fault-attacks on CRT-based RSA: Concrete results. In Proceedings of Austrochip'200\%, pages 61-67. Verlag der Technischen Universität, 2007. 